\title{
On the sensitivity of the ESPRIT algorithm to non-identical subarrays
}

\author{
A SWINDLEHURST ${ }^{1}$ and T KAILATH ${ }^{2}$ \\ ${ }^{1}$ Department of Electrical and Computer Engineering, Brigham Young \\ University, Provo, UT 84602, USA \\ ${ }^{2}$ Information Systems Laboratory, Stanford University, Stanford, CA \\ 94305, USA
}

\begin{abstract}
ESPRIT (estimation of signal parameters via rotational invariance techniques) is a recently introduced algorithm for narrowband directionof-arrival (DOA) estimation. Its principal advantage is that the DOA parameter estimates are obtained directly, without knowledge (and hence storage) of the array manifold and without computation or search of some spectral measure. This advantage is achieved by constraining the sensor array to be composed of two identical, translationally invariant subarrays. In this paper, we analyse the sensitivity of ESPRIT to the assumption that the subarrays are identical. The analysis is applicable to a wide variety of array errors, including non-identical angle-dependent and angle-independent gain and phase perturbations, errors in the locations of the subarray elements, and mutual coupling effects. A representative simulation example will be presented to validate the analysis and compare the performance degradation of ESPRIT with that of the MUSIC algorithm.
\end{abstract}

Keywords. Sensor array processing; direction-of-arrival estimation; perturbation analyses; antenna errors.

\section{Introduction}

Among the various high-resolution methods for narrowband direction-of-arrival (DOA) estimation proposed during the past decade, the ESPRIT (estimation of signal parameters via rotational invariance techniques) algorithm (Paulraj et al 1986; Roy et al 1986; Roy 1987; Roy \& Kailath 1989) has been one of the most promising. The algorithm achieves accurate DOA estimates without full knowledge of the antenna array response (no measurement or storage of calibration data is necessary), and without computation and search of some spectral measure. In addition, because ESPRIT has a very modular implementation involving only repeated low-order eigendecompositions, the algorithm is especially well-suited to real-time scenarios.

The advantages of ESPRIT result from a specific constraint on the physical geometry of the antenna array; namely, the array must be composed of two identical, 
translationally invariant subarrays. The individual elements of each subarray may have arbitrary directional gain and phase response, provided each has an identical twin in the companion subarray. In addition, the subarrays may overlap; i.e., an array element may be a member of each of the two subarrays. Such structures occur, for example, when implementing ESPRIT using a uniform linear array (ULA) of identical sensors.

In practice, it is of course impossible to construct any two sensors to be exactly identical. Any differences in the physical characteristics of the sensor (e.g., size, shape etc.) or in the receiver electronics behind each sensor will lead to differences in the nominal gain and phase response. Mutual coupling effects, especially between sensor pairs, will also result in subarray non-uniformity. Furthermore, it is impossible to guarantee that the subarrays have identical shape or that they be identically oriented (i.e., translations of one another). One would hope that when these subarray differences are slight, the resulting degradation in the parameter estimates will also be small. In any case, it is essential that the sensitivity of ESPRIT to these assumptions be quantified.

In this paper, such a sensitivity analysis is undertaken. The analysis is applicable to a wide variety of antenna errors, including angle-dependent and independent gain and phase perturbations, errors in the position and orientation of the subarrays, as well as mutual coupling effects. To isolate the effect of these errors on the DOA estimates, finite sample effects due to noise are neglected and expressions for the RMS estimation error are obtained in terms of the statistics of the perturbations. In contrast, several authors have considered the finite sample performance of ESPRIT (Rao \& Hari 1988, 1990; Ottersten et al 1989, 1990; Ottersten 1989), but their analyses assumed an unperturbed array response.

The organization of the paper is as follows: To begin with, a description is given in $\S 2$ of the narrowband DOA estimation problem and the ESPRIT solution. Most importantly, it is shown how ESPRIT may be formulated as a least-squares parameter estimation problem with a well-defined cost function. Several important models for antenna array errors are developed in $\S 3$, and their effect on the ESPRIT cost function is detailed in $\S 4$. In the latter section, a first-order perturbation analysis is used to link variations in the cost function with variations in the DOA estimates, allowing computation of the first and second moments of the estimation error. This analysis methodology follows closely that presented by Ottersten \& coworkers (Ottersten 1989; Ottersten et al 1989, 1990). A simulation example is presented in $\S 5$ to validate the analysis and illustrate the trade-offs associated with ULA subarray choices.

\section{The ESPRIT algorithm}

The ESPRIT algorithm (Paulraj et al 1986; Roy et al 1986; Roy 1987; Roy \& Kailath 1989 ) assumes that a given $M$-element sensor array is composed of two identical $m$-element subarrays separated by a fixed displacement vector $\Delta$. For subarrays that do not overlap (i.e., share elements), $M=2 m$, though in general, $M \leqslant 2 m$ since overlapping subarrays are allowed.

Sensor arrays with this type of configuration result in measurement models with a very special structure. Defining $\mathrm{a}(\theta)$ to be the array response vector for a narrowband emitter at DOA $\theta$, the vector response $z \in \mathbb{C}^{M}$ of the array at time $t$ (i.e., a snapshot) is given by the linear model

$$
\mathbf{z}(t)=\mathbf{A}_{e} \mathbf{s}(t)+\mathbf{n}(t),
$$


where $s(t) \in \mathbb{C}^{d}$ is the vector of signal amplitudes and phases at time $t, \mathbf{n}(t)$ is additive noise, and

$$
\mathbf{A}_{e} \stackrel{\text { der }}{=}\left[\mathbf{a}\left(\theta_{1}\right) \cdots \mathbf{a}\left(\theta_{d}\right)\right]
$$

The subscript $e$ denotes the fact that the array possesses the displacement invariance required by FSPRIT. Now let $\mathbf{J}_{0}$ and $\mathbf{J}_{1}$ be the $m \times M$ selection matrices that assign the elements of $\mathbf{z}(t)$ to subarrays 0 (the reference subarray) and 1 respectively, and define

$$
\mathbf{J}=\left[\begin{array}{l}
\mathbf{J}_{0} \\
\mathbf{J}_{1}
\end{array}\right] ; \quad \mathbf{A}=\mathbf{J}_{0} \mathbf{A}_{e}
$$

The basis of the ESPRIT algorithm is the observation that $\mathbf{J}_{1} \mathbf{A}_{e}=\mathbf{J}_{0} \mathbf{A}_{e} \Phi$ for the array geometry described above; i.e.,

$$
\mathbf{J A}_{e}=\left[\begin{array}{c}
\mathbf{A} \\
\mathbf{A \Phi}
\end{array}\right]
$$

where $\Phi$ is a unitary diagonal matrix with diagonal elements $\phi_{i}$ given by

$$
\phi_{i}=\exp \left\{-j 2 \pi \Delta \sin \theta_{i} / \lambda\right\}, \quad i=1, \ldots, d,
$$

$\lambda$ is the wavelength of the narrowband signal, and $\Delta=|\Delta|$. ESPRIT exploits the shift structure inherent in $\boldsymbol{A}_{e}$ to estimate $\boldsymbol{\Phi}$ and hence the values of DOA $\theta_{1}, \ldots, \theta_{d}$ without knowledge of $\mathbf{A}$.

As with other algorithms of this genre, ESPRIT requires that the $M$-dimensional complex vector space $\mathbb{C}^{M}$ of received snapshot vectors be separated into orthogonal subspaces, namely the signal subspace and the noise subspace. This is typically achieved via an eigendecomposition of the covariance matrix $\mathbf{R}=\mathscr{E}\left\{\mathbf{z}(t) \mathbf{z}^{*}(t)\right\}$. Assuming the noise is spatially white ${ }^{\dagger}$, we have

$$
\mathbf{R}=\mathbf{A}_{e} \mathbf{S} \mathbf{A}_{e}^{*}+\sigma^{2} \mathbf{I},
$$

where $\mathbf{S}$ is the covariance matrix of the emitter signals and $\sigma^{2}$ is the noise variance at each sensor. The covariance $\mathrm{S}$ is assumed to be of full rank $d$ (no unity correlated signals) and the columns of $\mathbf{A}$ are assumed to be linearly independent; i.e., the subarray manifold is assumed to be unambiguous.

The eigendecomposition of $\mathbf{R}$ has the form:

$$
\mathbf{R}=\sum_{i=1}^{M} \lambda_{i} \mathbf{e}_{i} \mathbf{e}_{i}^{*}=\mathbf{E}_{s} \Lambda_{s} \mathbf{E}_{s}^{*}+\sigma^{2} \mathbf{E}_{n} \mathbf{E}_{n}^{*},
$$

where $\mathbf{E}_{s}=\left[\mathbf{e}_{1} \vdots \cdots \vdots \mathbf{e}_{d}\right], \mathbf{E}_{n}=\left[\mathbf{e}_{d+1} \vdots \cdots: \mathbf{e}_{M}\right]$, and $\lambda_{1} \geqslant \cdots \geqslant \lambda_{d}>\lambda_{d+1}=\cdots=\lambda_{M}=\sigma^{2}$. The span of the $d$ eigenvectors $\mathbf{E}_{s}$ defines the signal subspace, and the orthogonal complement spanned by $\mathbf{E}_{n}$ defines the noise subspace. All subspace techniques are based on the observation that $\operatorname{span}\left\{\mathbf{E}_{s}\right\}=\operatorname{span}\left\{\mathbf{A}_{e}\right\}$. This implies that there exists a full rank $d \times d$ matrix $\mathbf{T}$ satisfying $\mathbf{E}_{s}=\mathbf{A}_{e} \mathbf{T}$, which in turn implies that $\mathbf{J E}_{s}=\mathbf{J} \mathbf{A}_{e} \mathbf{T}$.

\footnotetext{
${ }^{\dagger}$ As usual, the assumption of spatially white noise is not necessary; the extension to an arbitrary noise covariance $\sigma^{2} \Sigma$ is straightforward, provided $\Sigma$ is known.
} 
Consequently, using (2) and defining $\Psi \stackrel{\Psi \text { def }}{=} \mathbf{T}^{-1} \boldsymbol{\Phi} \mathbf{T}$,

$$
J_{E_{s}} \stackrel{\text { def }}{=}\left[\begin{array}{l}
\mathbf{E}_{0} \\
\mathbf{E}_{1}
\end{array}\right]=\left[\begin{array}{c}
\mathbf{A} \\
\mathbf{A \Phi}
\end{array}\right] \mathbf{T},
$$

which immediately leads to $\mathbf{E}_{1}=\mathbf{E}_{0} \mathbf{T}^{-1} \mathbf{\Phi} \mathbf{T}=\mathbf{E}_{0} \mathbf{\Psi}$. Thus, $\mathbf{E}_{0}$ and $\mathbf{E}_{1}$ have equivalent range spaces, and the parameters of interest are functions of the eigenvalues of the operator $\Psi$ that maps $\mathbf{E}_{0}$ onto $\mathbf{E}_{1}$.

In practice, the sample covariance ${ }^{\dagger} \hat{\mathbf{R}}$ defined by

$$
\hat{\mathbf{R}}=\frac{1}{N} \sum_{k=1}^{N} \mathbf{z}\left(t_{k}\right) \mathbf{z}^{*}\left(t_{k}\right)
$$

is used to estimate $\mathbf{R}$, and the corresponding estimates $\hat{\mathbf{E}}_{0}$ and $\hat{\mathbf{E}}_{1}$ of $\mathbf{E}_{0}$ and $\mathbf{E}_{1}$ will not exactly satisfy the relationship of (5). Hence, there is no operator that exactly maps the columns of $\hat{\mathbf{E}}_{0}$ onto those of $\hat{\mathbf{E}}_{1}$. Though a least-squares estimate of $\Psi$ may be easily obtained (Roy et al 1986; Kung et al 1986), since both $\hat{\mathbf{E}}_{0}$ and $\hat{\mathbf{E}}_{1}$ have errors, a total-least-squares (TLS) (Golub \& Van Loan 1984) estimate of $\Psi$ is more appropriate. As formulated in Roy (1987) and Roy \& Kailath (1989), the ESPRIT algorithm obtains a TLS estimate of $\boldsymbol{\Psi}$ from the following minimization problem:

Given subspace estimates $\hat{\mathbf{E}}_{0}$ and $\hat{\mathbf{E}}_{1}$, find a matrix

$$
\mathbf{F}_{\mathrm{def}}^{=}\left[\begin{array}{l}
\mathbf{F}_{0} \\
\mathbf{F}_{1}
\end{array}\right] \in \mathbb{C}^{2 d \times d},
$$

to minimize

subject to

$$
\hat{V}=\left\|\left[\hat{\mathbf{E}}_{0}: \hat{\mathbf{E}}_{1}\right] \mathbf{F}\right\|_{F}^{2}
$$

$$
\mathbf{F}^{*} \mathbf{F}=\mathbf{I}
$$

The procedure for solving this minimization problem is referred to as the ESPRIT algorithm. It is easily shown that $\mathbf{F}$ is the matrix of right singular vectors of $\left[\hat{\mathbf{E}}_{0}: \hat{\mathbf{E}}_{1}\right]$ corresponding to the $d$ smallest singular values, or equivalently the matrix of eigenvectors corresponding to the $d$ smallest eigenvalues of $\left[\hat{\mathbf{E}}_{0}: \hat{\mathbf{E}}_{1}\right]^{*}\left[\hat{\mathbf{E}}_{0}: \hat{\mathbf{E}}_{1}\right]$, and the estimate of $\Psi$ is given by

$$
\hat{\Psi}_{\mathrm{ES}}=-\mathbf{F}_{0} \mathbf{F}_{1}^{-1} \text {. }
$$

Thus, the ESPRIT algorithm essentially consists of performing an $M \times M$ eigendecomposition to get an estimated signal subspace from the sample covariance matrix of the measurements, followed by a $2 d \times 2 d$ eigendecomposition and a $d \times d$ eigendecomposition to get $\boldsymbol{\Phi}$ from which the parameter estimates are easily obtained (Roy 1987; Roy \& Kailath 1989).

\footnotetext{
${ }^{\dagger}$ In situations where there is insufficient data to form a full rank covariance, a singular value decomposition on the data matrix is computationally more efficient.
} 


\subsection{A subspace fitting formulation}

In appendix $A$, the equivalence of the problem formulated above and the more common TLS linear parameter estimation problem (Golub \& Van Loan 1984) is established. Therein, it is shown that the escimate of $\boldsymbol{\Psi}$ obtained from (7) through (10) is identical to the estimate obtained from the following minimization problem:

$$
\min _{\{A, \boldsymbol{\Phi}, T\}} \hat{V}=\min _{\{A, \boldsymbol{\Phi}, T\}}\left\|\left[\begin{array}{l}
\hat{\mathbf{E}}_{0} \\
\hat{\mathbf{E}}_{1}
\end{array}\right]-\left[\begin{array}{c}
\mathbf{A} \\
\mathbf{A \Phi}
\end{array}\right] \mathbf{T}\right\|_{F}^{2} .
$$

Clearly, the ESPRIT algorithm is implementing a least-squares fit of the model described by (5) to the subspace estimate $\mathbf{J W}_{s}$. This subspace-fitting formulation of the problem is more appealing than the one given in (7) through (10) since it more clearly illustrates the problem being solved.

The minimization of $(11)$ is separable in the variable $T$, and as such can be rewritten in the more compact form

where

$$
\begin{aligned}
\hat{\boldsymbol{\eta}}=\arg \min _{\boldsymbol{\eta}} \hat{V} & =\underset{\boldsymbol{\eta}}{\arg \min }\left\|\mathbf{P}_{\mathbf{G}}^{\perp}(\boldsymbol{\eta}) \mathbf{J} \hat{\mathbf{E}}_{\boldsymbol{s}}\right\|_{\boldsymbol{F}}^{2} \\
& =\operatorname{Tr}\left(\mathbf{P}_{G}^{\perp} \mathbf{J} \hat{\mathbf{E}}_{s} \hat{\mathbf{E}}_{s}^{*} \mathbf{J}^{T}\right),
\end{aligned}
$$

$$
\mathbf{G} \stackrel{\text { def }}{=} \mathbf{J A}_{e}=\left[\begin{array}{c}
\mathbf{A} \\
\mathbf{A} \boldsymbol{\Phi}
\end{array}\right]
$$

and $\boldsymbol{\eta}$ is a vector containing the parameters estimated by the ESPRIT algorithm (i.e., the elements of $\mathbf{A}$ and $\boldsymbol{\Phi}$ ). In Ottersten et al (1990) it is shown that the following choice for $\boldsymbol{\eta}$ uniquely parameterizes the ESPRIT problem:

$$
\boldsymbol{\eta}=\left[\begin{array}{c}
a_{3 t} \\
a_{3 m} \\
\boldsymbol{\rho} \\
\boldsymbol{\theta}
\end{array}\right],
$$

where $a_{\varkappa_{t}}=\mathscr{R} e\{\operatorname{vec}(\tilde{\mathbf{I}} \mathbf{A})\}, a_{g_{m}}=\mathscr{I}_{m}\{\operatorname{vec}(\tilde{\mathbf{I}} \mathbf{A})\}, \quad \boldsymbol{\theta}=\left[\theta_{1}, \ldots, \theta_{d}\right]^{T}, \boldsymbol{\rho}=\left[\rho_{1}, \ldots, \rho_{d}\right]^{T}$ contains the magnitudes of the diagonal elements of $\Phi$, and $\widetilde{\mathbf{I}}=\left[\mathbf{0}_{(m-1) \times 1} \mathbf{I}\right]$ picks out all but the first row of the matrix to its right. The vector $\rho$ must be included as an estimated parameter since ESPRIT does not constrain the diagonal elements of $\Phi$ to lie on the unit circle. Also, since scaling the columns of $\mathbf{G}$ does not affect the parameter estimates, uniqueness is maintained by fixing the first row of $\mathbf{A}$ (as a row of ones, for example) and only estimating $a_{3 t}$ and $a_{s m}$, the elements of $\mathbf{A}$ in rows 2 through $m$.

Thus, ESPRIT can be viewed as simply a set of rules for mapping a subspace estimate $\widehat{\mathbf{E}}_{s} \in \mathbb{C}^{m \times d}$ into a unique estimate $\hat{\boldsymbol{\eta}} \in \mathbb{R}^{2 m d}$ from the parameter space. In the next section, we develop statistical models $\tilde{\mathbf{A}}_{e}$ for errors to the nominal antenna array response $\mathbf{A}_{e}$, and show how these errors translate into a model for the perturbed subspace $\hat{\mathbf{E}}_{\boldsymbol{s}}$. Ultimately, we are interested in the statistics of the image of $\hat{\mathbf{E}}_{s}$ in parameter space after it passes through the nonlinear ESPRIT transformation. 


\section{Error models}

Errors in the ESPRIT DOA estimates can arise from any of a number of sources. The most important of these include:

(i) finite sample effects;

(ii) an imprecisely known noise covariance;

(iii) a perturbed array manifold.

Finite sample effects occur because we must use the covariance estimate of (6). For finite $N$, the signais and noise have not had "time" to decorrelate, and the noise covariance has not yet converged to its limiting value. When $N$ is large or the SNR is high, finite sample effects may be neglected. There are in fact many applications for which the limiting factor in performance is not due to finite sample effects, but rather to the model errors of items (ii) and (iii). In this analysis, we will focus only on item (iii).

Since ESPRIT requires two identical, translationally invariant antenna subarrays, and does not assume knowledge of a fully calibrated array manifold, one might think it most convenient to consider only one of the subarrays as having any error associated with it. In other words, one might assume that if $\mathbf{A}$ represents the response of the first subarray (whatever it is), then $\mathbf{A \Phi}+\tilde{\mathbf{A}}$ might represent the response of the second, where $\tilde{\mathbf{A}}$ is some additive disturbance. However, even though the algorithm does not require knowledge of $\mathbf{A}$, the resulting error expressions for the parameters will be a function of the nominal subarray response. To evaluate the performance of ESPRIT for a given scenario, it must thus be assumed that the full array response is available. Because of this fact, it is more convenient to consider errors which directly affect the calibrated manifold, using for example the model $\hat{\mathbf{A}}_{e}=\mathbf{A}_{e}+\tilde{\mathbf{A}}_{e}$. Under this model, since $\mathbf{J}_{1} \hat{\mathbf{A}}_{e} \neq \mathbf{J}_{0} \hat{\mathbf{A}}_{e} \boldsymbol{\Phi}$ in general, the quantity $\mathbf{J} \tilde{\mathbf{A}}_{e}$ represents deviations of the array response from the shift invariant structure of (5).

To isolate the effects of array perturbations on the DOA estimates, it is assumed that the finite sample effects due to additive noise are negligible and that an exact measurement of the perturbed covariance $\hat{\mathbf{R}}$ is available. Using an additive model for the array error, $\hat{\mathbf{R}}$ may be written as

$$
\hat{\mathbf{R}}=\hat{\mathbf{A}}_{e} \mathbf{S} \hat{\mathbf{A}}_{e}^{*}+\sigma^{2} \mathbf{I}=\left(\mathbf{A}_{e}+\tilde{\mathbf{A}}_{e}\right) \mathbf{S}\left(\mathbf{A}_{e}+\tilde{\mathbf{A}}_{e}\right)^{*}+\sigma^{2} \mathbf{I}
$$

The matrix $\tilde{\mathbf{A}}_{e}$ represents the error in the nominal array response, and incorporates the effects of imprecisely known sensor locations, perturbations in the antenna amplitude and phase patterns, and mutual coupling effects. Each of the columns of $\hat{\mathbf{A}}_{e}$ will be denoted in a similar way: $\hat{\mathbf{a}}\left(\theta_{i}\right)=\mathbf{a}\left(\theta_{i}\right)+\tilde{\mathbf{a}}\left(\theta_{i}\right), \quad i=1, \ldots, d$.

\subsection{Antenna array perturbations}

An example of a perturbed sensor array is depicted in figure 1. The array is nominally assumed to be composed of uniformly spaced identical elements; i.e., each sensor is assumed to have identical response, the signal conditioning electronics (e.g., filter gain and phase response, automatic gain controls (AGC), etc.) are assumed to perform identically, and the analog-to-digital (A/D) converters are assumed to be synchronized. However, as shown in the figure, the sensors are not identical (their beampatterns are different), and their positions are not uniform. In addition, the filter and AGC 


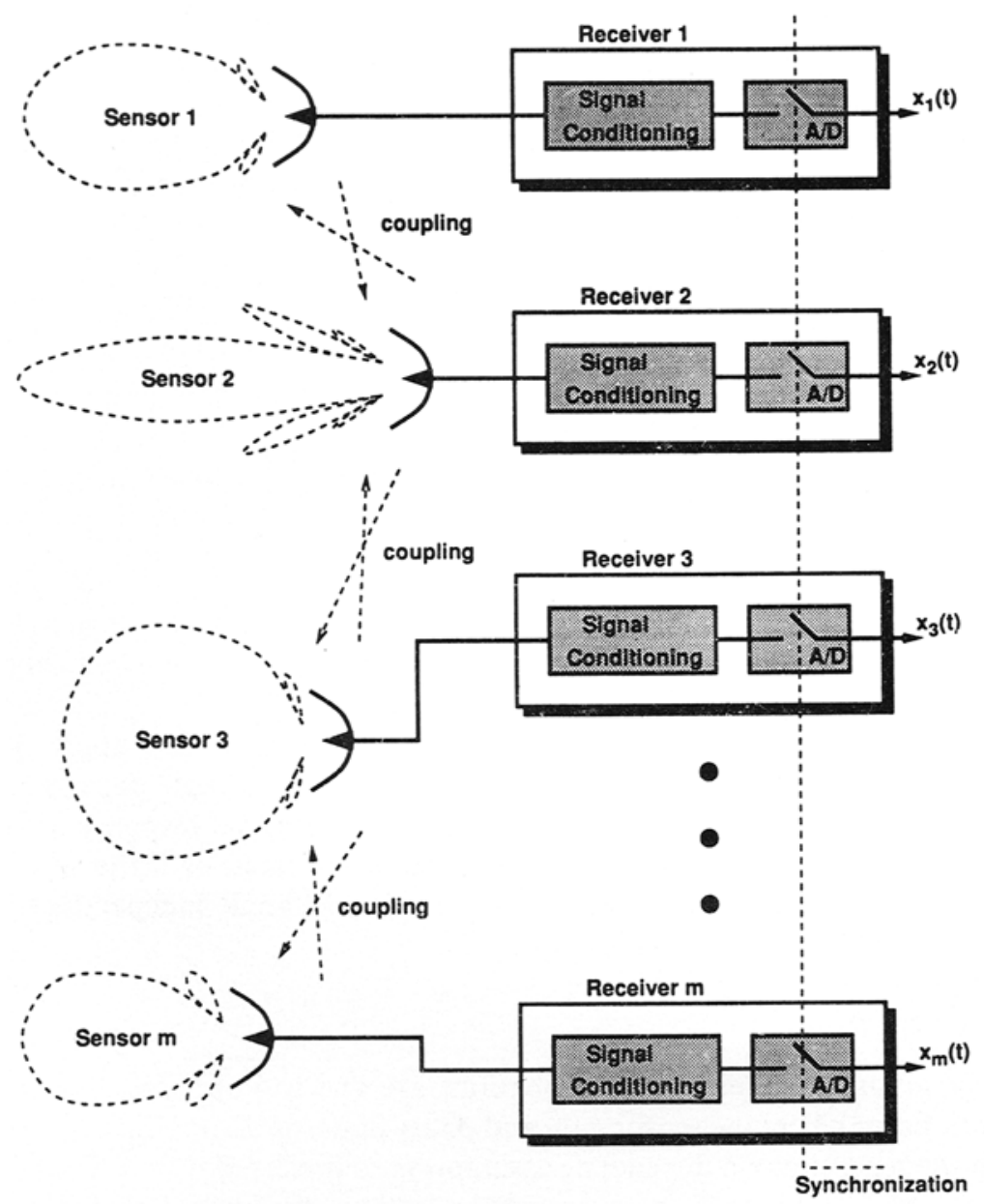

Figure 1. A sample perturbed array.

characteristics will not be uniform from receiver to receiver, the A/D converters will not be exactly in phase, and there may be uncalibrated or non-uniform mutual coupling present ${ }^{\dagger}$. All of these factors combine in varying degrees to produce the array perturbation $\tilde{\mathbf{A}}_{e}$.

There are a variety of models that could be used to describe $\tilde{\mathbf{A}}_{e}$. A particularly simple approach is to assume that the columns of $\tilde{\mathbf{A}}_{e}$ are independent zero-mean complex Gaussian random vectors with known covariance:

$$
\tilde{\mathbf{a}}\left(\theta_{i}\right) \sim \mathbb{C} \mathcal{H}\left(0, \mathbf{B}_{i}\right), \quad \mathscr{E}\left\{\tilde{\mathbf{a}}\left(\theta_{i}\right) \tilde{\mathbf{a}}^{T}\left(\theta_{i}\right)\right\}=0, \quad i=1, \ldots, d .
$$

If the errors are independent from sensor-to-sensor, $\mathbf{B}_{i}$ is clearly diagonal. Off-diagonal terms indicate sensor-to-sensor correlations that result, for example, if there are uncalibrated mutual coupling effects, or if some sensors tend to perturb uniformly (such as identical or adjacent elements). Though convenient for such performance

\footnotetext{
${ }^{\dagger}$ Mutual coupling occurs when a nominally passive collector acts as a transmitter and reradiates some of its received energy.
} 
comparisons, this simple model is not as ideal for performance analysis since, for example, it is difficult to control gain and phase errors independently.

To see how a more general model may be obtained, suppose that $a_{k}$ and $e^{j \phi_{k}}$ are respectively the nominal gain and phase response of the $k$ th sensor to a signal from direction $\theta$; i.e., $\mathbf{a}_{k}(\theta)=a_{k} e^{j \phi_{k}}$. The perturbed response $\hat{\mathbf{a}}_{\boldsymbol{k}}(\theta)=\mathbf{a}_{k}(\theta)+\tilde{\mathbf{a}}_{k}(\theta)$ with separate gain and phase errors $\tilde{a}_{k}$ and $\tilde{\phi}_{k}$ may be written as

$$
\begin{aligned}
\hat{\mathbf{a}}_{k}(\theta) & =\left(a_{k}+\tilde{a}_{k}\right) e^{j\left(\phi_{k}+\tilde{\phi}_{k}\right)} \\
& =a_{k} e^{j \phi_{k}}\left[1+\left(\tilde{a}_{k} / a_{k}\right)\right] e^{j \tilde{\phi}_{k}} \\
& =\mathbf{a}_{k}(\theta)+\gamma_{k}(\theta) \mathbf{a}_{k}(\theta),
\end{aligned}
$$

where $\gamma_{k}(\theta) \stackrel{\text { def }}{=}\left(1+\tilde{a}_{k} / a_{k}\right) e^{j \tilde{\phi}_{k}}-1$. The $m \times d$ matrix $\tilde{\mathbf{A}}_{e}$ is thus described by the equation

$$
\tilde{\mathbf{A}}_{e}=\left[\boldsymbol{\Gamma}_{1} \mathbf{a}\left(\theta_{1}\right): \boldsymbol{\Gamma}_{2} \mathbf{a}\left(\theta_{2}\right) \vdots \ldots: \boldsymbol{\Gamma}_{d} \mathbf{a}\left(\theta_{d}\right)\right],
$$

where $\Gamma_{i}=\operatorname{diag}\left\{\gamma_{1}\left(\theta_{i}\right), \ldots, \gamma_{m}\left(\theta_{i}\right)\right\}$. Though for simplicity we have written $\boldsymbol{\Gamma}_{i}$ as a diagonal matrix, the off-diagonal elements will be non-zero for cases involving unmodeled mutual coupling ( $c f$. Weiss \& Friedlander 1988).

The models of (15) and (17) are general in the sense that they apply to both angle-independent and angle-dependent sensor errors. For the angle-dependent case, $\mathbf{B}_{i} \neq \mathbf{B}_{k}$ and $\Gamma_{i} \neq \Gamma_{k}$ for $i \neq k$. If the deviations from the nominal response are due to bulk delay and gain errors in the antenna receiver electronics, or if the sources are grouped closely in angle, the errors may be assumed to be angle-independent. Under this assumption, (17) may be written as ${ }^{\dagger}$

$$
\tilde{\mathbf{A}}_{e}=\Gamma \mathbf{A}_{e} .
$$

However, in situations where the perturbations are due to imprecise knowledge of sensor locations, or where the sensor gain and phase patterns do not distort uniformly in $\theta$, the more general angle-dependent assumption is preferred.

In practice, the response of a given sensor is typically known to within some tolerance in gain and phase that accounts for variations in the construction of the sensor and the conditions under which it is to operate. This tolerance may be specified as limits above and below some nominal response, or as an expected deviation around the nominal. Consequently, as mentioned in the previous section, we will assume in this analysis that $\tilde{\mathbf{A}}_{e}$ is specified in probabilistic terms (e.g., the mean and variance of the elements of $\tilde{\mathbf{A}}_{e}$ are assumed known). This assumption has already been implicitly made in the model of (15). In this framework, one may think of the sensor array as one realization from the probability space of arrays specified by $\mathbf{A}_{e}$ and the distribution of $\tilde{\mathbf{A}}_{e}$. As such, when simulation studies are conducted to verify this analysis, Monte Carlo trials will be performed over a large number of arrays "drawn" from the distribution specified by $\boldsymbol{A}_{e}$ and $\tilde{\mathbf{A}}_{\boldsymbol{e}}$.

\subsection{Signal subspace effects}

Since ESPRIT is based on structure inherent in the eigenvectors of $\mathbf{R}$ which span the signal subspace, we are primarily interested in the effects of $\tilde{\mathbf{A}}_{e}$ on $\hat{\mathbf{E}}_{s}$. To establish a

\footnotetext{
t The error model described by $(18)$ is only valid when the gain response of each sensor is also independent of $\theta$.
} 
link between the array errors of (14) and the subspace estimate $\hat{\mathbf{E}}_{s}$, let $\hat{\mathbf{E}}_{s}=\mathbf{E}_{s}+\tilde{\mathbf{E}}_{s}$ and examine the eigendecomposition

$$
\hat{\mathbf{R}} \hat{\mathbf{E}}_{s}=\hat{\mathbf{E}}_{s}\left(\Lambda_{s}+\tilde{\Lambda}_{s}\right)
$$

where $\Lambda_{s}$ and $\tilde{\Lambda}_{s}$ represent the nominal and perturbed signal eigenvalues, respectively. Expanding (19) using the model of (14) and eliminating second-order error terms (e.g., terms of order $O\left(\left\|\tilde{\mathbf{A}}_{e}\right\|^{2}\right), O\left(\left\|\tilde{\mathbf{A}}_{e}\right\|\left\|\tilde{\mathbf{E}}_{s}\right\|\right)$, etc.) leads to the following first-order approximation:

$$
\left(\tilde{\mathbf{A}}_{e} \mathbf{S A} \mathbf{A}_{e}^{*}+\mathbf{A}_{e} \mathbf{S} \tilde{\mathbf{A}}_{e}^{*}\right) \mathbf{E}_{s}+\mathbf{R} \tilde{\mathbf{E}}_{s}=\mathbf{E}_{s} \tilde{\mathbf{\Lambda}}_{s}+\tilde{\mathbf{E}}_{s} \mathbf{\Lambda}_{s} .
$$

After multiplying on the left by $\mathbf{P}_{G}^{\perp} \mathbf{J}$, a few simple algebraic manipulations yield the following:

$$
\tilde{\mathbf{E}}_{s}^{*} \mathbf{J}^{T} \mathbf{P}_{G}^{\perp}=\tilde{\Lambda}^{-1} \mathbf{E}_{s}^{*} \mathbf{A}_{e} \mathbf{S} \tilde{A}_{e}^{*} \mathbf{J}^{T} \mathbf{P}_{G}^{\perp},
$$

where $\tilde{\boldsymbol{\Lambda}} \stackrel{\text { def }}{=} \boldsymbol{\Lambda}_{s}-\sigma^{2}$ I. Equation (20) relates the physical antenna perturbation $\tilde{\mathbf{A}}_{\boldsymbol{e}}$ with the component of the perturbed signal subspace projected into the true noise subspace (the only component that can lead to estimation error). This relationship is the key to linking the statistics of $\tilde{\mathbf{A}}_{e}$ with those of the DOA estimation error, as will be outlined in the next section.

\section{ESPRIT error analysis}

In this section, we will obtain a general expression for the covariance of the estimation error $^{+}$

$$
\mathbf{C}_{\mathrm{ES}} \stackrel{\text { def }}{=} \mathscr{E}\left\{\left(\hat{\boldsymbol{\eta}}-\boldsymbol{\eta}_{0}\right)\left(\hat{\boldsymbol{\eta}}-\boldsymbol{\eta}_{\mathbf{0}}\right)^{T}\right\}
$$

in terms of the statistics of the array perturbations $\tilde{\mathbf{A}}_{\boldsymbol{e}}$. Following the first order analysis in Ottersten et al $(1989,1990)$, we expand the ESPRIT cost function around the minimizing parameter estimate $\hat{\boldsymbol{\eta}}$ :

$$
\begin{aligned}
0=\hat{V}^{\prime}(\hat{\boldsymbol{\eta}}) & =\hat{V}^{\prime}\left(\boldsymbol{\eta}_{0}\right)+\hat{V}^{\prime \prime}\left(\boldsymbol{\eta}_{0}\right)\left(\hat{\boldsymbol{\eta}}-\boldsymbol{\eta}_{0}\right) \\
& \simeq \hat{V}^{\prime}\left(\boldsymbol{\eta}_{0}\right)+V^{\prime \prime}\left(\boldsymbol{\eta}_{0}\right)\left(\hat{\boldsymbol{\eta}}-\boldsymbol{\eta}_{0}\right),
\end{aligned}
$$

where we have used the notation

$$
\hat{V}^{\prime}\left(\boldsymbol{\eta}_{0}\right)=\left.\frac{\partial \hat{V}(\boldsymbol{\eta})}{\partial \boldsymbol{\eta}}\right|_{\boldsymbol{\eta}=\boldsymbol{\eta}_{0}}, \quad V^{\prime \prime}\left(\boldsymbol{\eta}_{0}\right)=\left.\frac{\partial V(\boldsymbol{\eta})}{\partial \boldsymbol{\eta} \partial \boldsymbol{\eta}^{T}}\right|_{\boldsymbol{\eta}=\boldsymbol{\eta}_{0}},
$$

and where $V$ represents $\hat{V}$ evaluated using $\mathbf{E}_{s}$ instead of $\hat{\mathbf{E}}_{s}$. Replacing $\hat{V}^{\prime \prime}\left(\boldsymbol{\eta}_{0}\right)$ with $V^{\prime \prime}\left(\eta_{0}\right)$ in the approximate equality of $(21)$ is justified to first order since it is multiplied by the error $\hat{\boldsymbol{\eta}}-\boldsymbol{\eta}_{0}$ which is assumed to be relatively small. Since we are exclusively interested in evaluating the cost function derivatives at the true parameter vector $\eta_{0}$, in the sequel we will use the simplifying notation $V^{\prime \prime}=V^{\prime \prime}\left(\eta_{0}\right)$ and $\hat{V}^{\prime}=\hat{V}^{\prime}\left(\eta_{0}\right)$.

\footnotetext{
+ We will consider only the second moment of the error since to first order, zero-mean array perturbations produce no estimate bias. This is shown in appendix $B$.
} 
Solving (21) for the error in the parameter vector gives

$$
\hat{\boldsymbol{\eta}}-\eta_{0} \simeq\left(V^{\prime \prime}\right)^{-1} \hat{V}^{\prime},
$$

so $\mathbf{C}_{\mathrm{ES}}$ may be expressed as

$$
\mathbf{C}_{\mathrm{ES}}=\left(V^{\prime \prime}\right)^{-1} \mathbf{Q}\left(V^{\prime \prime}\right)^{-1}
$$

where $\mathbf{Q} \stackrel{\text { def }}{=} \mathscr{E}\left\{\left(\hat{V}^{\prime}\right)\left(\hat{V}^{\prime}\right)^{T}\right\}$. It remains now to specify the matrices $V^{\prime \prime}$ and $\mathbf{Q}$ in terms of known quantities. An expression for $V^{\prime \prime}$ was obtained in Ottersten et al (1989, 1990), where it was shown that the $i, k$ th element of this matrix is given by

$$
\begin{aligned}
V_{i k}^{\prime \prime} & =-2 \mathscr{R e}\left[\operatorname{Tr}\left(G_{i}^{*} \mathbf{P}_{G}^{\perp} \mathbf{G}_{k} G^{\dagger} \mathbf{J} E_{s} \mathbf{E}_{s}^{*} \mathbf{J}^{T} \mathbf{G}^{\dagger *}\right)\right] \\
& =-2 \mathscr{R e}\left[\operatorname{Tr}\left(\mathbf{G}_{i}^{*} \mathbf{P}_{G}^{\perp} G_{k}\left(A_{e}^{*} \mathbf{A}_{e}\right)^{-1}\right)\right] .
\end{aligned}
$$

The matrix $\mathbf{G}_{\boldsymbol{i}}$ is defined here to be

$$
\left.\mathbf{G}_{i} \stackrel{\text { del }}{=} \frac{\partial \mathbf{G}}{\partial \eta_{i}}\right|_{\eta=\eta_{0}},
$$

where $\eta_{i}$ denotes the $i$ th element of $\boldsymbol{\eta}$.

To complete the definition of $\mathbf{C}_{\mathrm{ES}}$, it is shown in appendix $\mathrm{B}$ that the $i, k$ th element of $\mathbf{Q}$ is given by

where

$$
\mathbf{Q}_{i k}=\mathbf{E}\left\{\hat{V}_{i}^{\prime} \hat{V}_{k}^{\prime}\right\}=2 \mathscr{R} e\left[\sum_{p=1}^{d} \sum_{q=1}^{d_{1}} \mathbf{Y}_{p}^{(i)} \mathbf{C}_{a, 1}^{p q} \mathbf{Y}_{q}^{(k) T}+\mathbf{Y}_{p}^{(i)} \mathbf{C}_{a, 2}^{p q} \mathbf{Y}_{q}^{(k)^{*}}\right]
$$

$$
\begin{aligned}
& \hat{\boldsymbol{V}}_{i}^{\prime \text { def }}=\left.\frac{\partial \hat{V}}{\partial \boldsymbol{\eta}_{i}}\right|_{\eta=\eta_{0}} \\
& \mathbf{Y}^{(i)}=\left(\mathbf{A}_{e}^{*} \mathbf{A}_{e}\right)^{-1} \mathbf{G}_{i}^{*} \mathbf{P}_{G}^{\perp} \mathbf{J} \\
& \mathbf{C}_{a, 1}^{p q}=\mathscr{E}\left\{\tilde{\mathbf{a}}\left(\theta_{p}\right) \tilde{\mathbf{a}}^{T}\left(\theta_{q}\right)\right\} \\
& \mathbf{C}_{a, 2}^{p q}=\mathscr{E}\left\{\tilde{\mathbf{a}}\left(\theta_{p}\right) \tilde{\mathbf{a}}^{*}\left(\theta_{q}\right)\right\},
\end{aligned}
$$

and $\mathbf{Y}_{p}^{(i)}$ denotes the $p$ th row of $\mathbf{Y}^{(i)}$. Expressions for the derivative terms $\mathbf{G}_{i}$ needed in computing $V^{\prime \prime}$ and $\mathbf{Q}$ are also detailed in appendix $\mathrm{B}$. With expressions for $V^{\prime \prime}$ and $\mathbf{Q}$ now in hand, we see that the covariance of the DOA estimation error $\mathbf{C}_{E S}$ may be determined for a particular scenario provided that the covariances of the array perturbations $C_{a, 1}^{p q}$ and $C_{a, 2}^{p q}$ are known. In Swindlehurst \& Kailath (1990), the form of $\mathbf{C}_{a, 1}^{p q}$ and $\mathbf{C}_{a, 2}^{p q}$ is examined for several special cases involving various gain and phase errors, sensor positioning errors, and mutual coupling effects.

A relatively simple expression for $\mathbf{Q}$ results when the Gaussian error model of (15) is assumed. It is shown in appendix $B$ that if the array errors are independent and identically distributed from angle to angle, i.e., if $\mathscr{E}\left\{\tilde{\mathbf{a}}\left(\theta_{i}\right) \tilde{\mathbf{a}}^{*}\left(\theta_{k}\right)\right\}=0$ for $i \neq k$ and $\mathbf{B}_{i}=\mathbf{B}$ for $i=1, \ldots, d$, then (23) simplifies to

$$
\mathbf{Q}_{i k}=2 \mathscr{R e}\left[\operatorname{Tr}\left(\mathbf{G}_{i}^{*} \mathbf{P}_{G}^{\perp} \mathbf{J B} \mathbf{J}^{T} \mathbf{P}_{G}^{\perp} \mathbf{G}_{k}\left(\mathbf{A}_{e}^{*} \mathbf{A}_{e}\right)^{-2}\right)\right] .
$$

To validate the first-order analysis of this section, this easily tractable model is assumed for the simulation example of the next section. 


\section{A simulation example}

In this example, we consider the performance of ESPRIT for several different ULA subarray choices. A 10-element ULA with $\lambda / 2$ interelement spacing was assumed and two emitters were simulated, one at $0^{\circ}$ broadside and the other at $7^{\circ}$. The signal-to-noise ratio was 12 and $20 \mathrm{~dB}$ for the $0^{\circ}$ and $7^{\circ}$ sources, respectively, and they were assumed to be $90 \%$ correlated with $0^{\circ}$ correlation phase. The nominal gain of all sensors was assumed to be unity in the direction of the impinging signals, and the noise was assumed to be spatially white with unit variance.

To simulate the effects of sensor errors, the exact perturbed covariance $\hat{\mathbf{R}}$ was generated in each case using the error-free covariance $\mathbf{R}$ and the distribution of the perturbation. For simplicity's sake, the Gaussian perturbation of (15) with error covariance $\mathbf{B}_{1}=\mathbf{B}_{2}=\gamma^{2} \mathbf{I}$ was used in this case to generate the array errors. The perturbation amplitude $\gamma$ was varied from 0.0001 to 0.4 in several steps, and 1000 trials were conducted for each step. The sample standard deviation of the DOA estimates was then calculated and compared to that predicted by the corresponding theoretical expressions. The results of this simulation are plotted in figure 2 for the case of the broadside source.

Each of the three implementations of ESPRIT corresponds to a different choice for $\mathbf{J}$, the subarray selection matrix. The variable $\Delta$ indicates the distance between the subarrays in terms of the wavelength $\lambda$. As the name implies, two interleaved ESPRIT subarrays are obtained by separating the even- and odd-numbered elements of the ULA. For overlapping subarrays, the first $m-k$ elements are grouped in one subarray, and the last $m-k$ elements in another. In the figure, $\Delta=\lambda / 2$ and $\Delta=3 \lambda / 2$ correspond to $k=1$ and $k=3$ respectively. Note also that, for purposes of comparison, the performance of the root-MUSIC algorithm (e.g., see.Roy 1987; Schmidt 1981; Rao \& Hari 1989 ) is also included. For the case of array perturbations considered here, theoretical

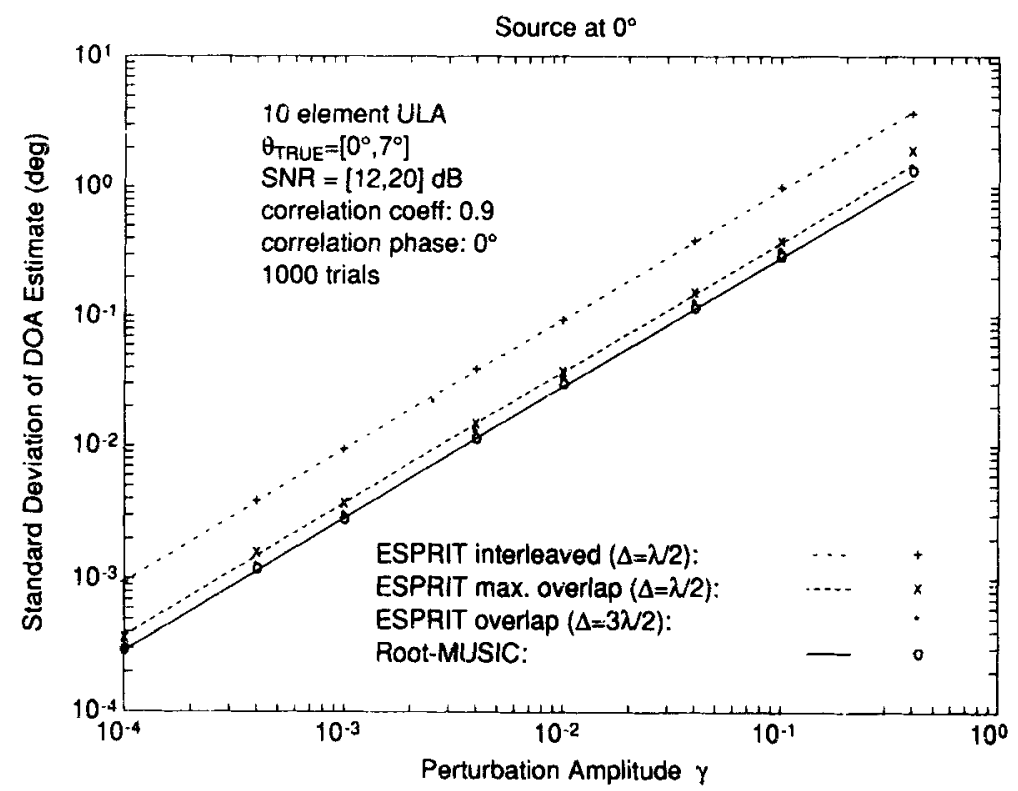

Figure 2. Actual and predicted DOA errors $v s . \gamma$. The connected lines in the plot denote predicted performance, while the symbols ' $\times \mathrm{O}_{*}$ " indicate the results of the simulations. 
expressions for the root-MUSIC estimation error can be found in Swindlehurst \& Kailath (1990) and Swindlehurst et al (1989).

There are several important conclusions that can be drawn from this example. First, there is excellent agreement between predicted and measured algorithm performance. This is true even at the relatively large value of $y=0.4$, which corresponds to a standard deviation in gain of 0.4 (relative to unity gain) and a standard deviation in phase of roughly $24^{\circ}$. Second, ESPRIT tends to degrade quite gracefully as the degree of perturbation increases. For example, at $\gamma=0.1$ corresponding to a gain and phase standard deviation of 0.1 and $6^{\circ}$ (which is well beyond the tolerance of many commercial sensing devices), the standard deviation of the ESPRIT DOA estimates varies from only 0.2 to 1 degree, depending on the subarray separation. Performance improves as $\Delta$ increases because of the increased baseline between the subarrays. However, at larger values of $\Delta$, the diminished subarray size begins to play against this advantage and the DOA error will increase.

Another interesting observation is that the standard deviation of the estimates is linear in the perturbation amplitude $\gamma$; i.e., an order of magnitude increase in $\gamma$ results in a corresponding order of magnitude increase in the DOA estimates. This is true of both ESPRIT and root-MUSIC. Note also that, of the four algorithms implemented, root-MUSIC was the least sensitive to this type of sensor error, though ESPRIT with $\Delta=3 \lambda / 2$ is only slightly worse. Based on results obtained in Swindlehurst \& Kailath (1990), it is the authors' conjecture that root-MUSIC (and MUSIC as well) actually achieve the Cramér-Rao lower bound for this problem (i.e., infinite data, independent Gaussian array perturbations), and hence that no other algorithm will have lower estimation error variance. If this is indeed the case, we see that the much more computationally efficient ESPRIT algorithm is in this case able to achieve near optimal performance.

\section{Conclusions}

An analysis of the sensitivity of the ESPRIT algorithm to antenna array perturbations has been presented in this paper. The analysis methodology is quite general, and may be applied to cases where the subarray mismatch is due to non-identical sensor gain and phase responses, non-identical subarray shape and orientation, and mutual coupling effects. A general model incorporating these types of sensor errors was developed and, using first-order perturbation arguments, the resulting error in the signal subspace was linked with the error in the ESPRIT cost function, and in turn with the ESPRIT DOA estimates themselves. In particular, a closed-form expression was obtained for the DOA estimation error variance in terms of the variance of the array perturbations. The validity of the analysis was demonstrated via a simple simulation example wherein the performance of ESPRIT was compared with that of the popular root-MUSIC algorithm.

This work was supported in part by the SDIO/IST Program managed by the Office of Naval Research under Contract N00014-85-K-0550, ARO/SDI and by the Joint 
Services Program at Stanford University (US Army, US Navy, US Air Force) under Contract DAAL03-88-C-0011, and by grants from Rockwell International and the General Electric Company.

Appendix A. Equivalence of several forms of the ESPRIT problem.

The minimization problem posed in (7) through (10) is not the most common statement of the TLS linear parameter estimation problem. In Golub \& Van Loan (1984), for example, the problem is posed in the following manner:

Given $\mathbf{C}, \mathbf{D} \in \mathbb{C}^{m \times d}, m>d$, find $\mathbf{R}_{C}$ and $\mathbf{R}_{D}$ of minimum Frobenius norm, and $\mathbf{X}$ such that

$$
\left[\mathbf{C}+\mathbf{R}_{\mathbf{C}}\right] \mathbf{X}=\mathbf{D}+\mathbf{R}_{\boldsymbol{D}}
$$

The coninection with the original formulations of ESPRIT given in $\$ 2$ can be made by noting that $\mathbf{R}_{C}$ and $\mathbf{R}_{D}$ represent errors in the subspace estimates $\hat{\mathbf{E}}_{0}=\mathbf{C}$ and $\hat{\mathbf{E}}_{1}=\mathbf{D}$ respectively, and that $\mathbf{X}$ is the operator $\boldsymbol{\Psi}$. Using these variable definitions and theorem 12.3-1 of Golub \& Van Loan (1984), the solution $\hat{\mathbf{\Psi}}_{\mathrm{TLS}}$ of the above minimization problem is given by

where

$$
\hat{\boldsymbol{\Psi}}_{\mathrm{TLS}}=-\mathbf{V}_{12} \mathbf{V}_{22}^{-1}
$$

$$
\mathbf{V}=\left[\begin{array}{ll}
\mathbf{V}_{11} & \mathbf{V}_{12} \\
\mathbf{V}_{21} & \mathbf{V}_{22}
\end{array}\right]
$$

is the matrix of right singular vectors obtained from the following singular value decomposition:

$$
\overline{\mathbf{E}} \stackrel{\text { def }}{=}\left[\hat{\mathbf{E}}_{0}: \hat{\mathbf{E}}_{1}\right]=\mathbf{U} \mathbf{\Sigma} \mathbf{V}^{*}
$$

In Roy (1987) and Roy \& Kailath (1989) it is shown that the ESPRIT estimate of $\Psi$ for the formulation of the problem in equations (7) through (10) is given by $\hat{\mathbf{\Psi}}_{\mathrm{ES}}=-\mathbf{E}_{12} \mathbf{E}_{22}^{-1}$, where

$$
\overline{\mathbf{E}}^{*} \overline{\mathbf{E}}=\left[\begin{array}{ll}
\mathbf{E}_{11} & \mathbf{E}_{12} \\
\mathbf{E}_{21} & \mathbf{E}_{22}
\end{array}\right] \Lambda\left[\begin{array}{ll}
\mathbf{E}_{11} & \mathbf{E}_{12} \\
\mathbf{E}_{21} & \mathbf{E}_{22}
\end{array}\right]^{*}
$$

is the eigendecomposition of $\overline{\mathbf{E}}^{*} \overline{\mathbf{E}}$. The equivalence of the right singular vectors of $\overline{\mathbf{E}}$ and the eigenvectors of $\overline{\mathbf{E}}^{*} \overline{\mathbf{E}}$ establishes the equivalence of $\hat{\mathbf{\Psi}}_{\mathrm{TLS}}$ and $\hat{\mathbf{\Psi}}_{\mathrm{ES}}$.

The equivalence of the operator $\Psi$ obtained from (7) through (10), and that obtained from (11) will now be established. Using standard properties of the trace operator, the minimization of (8) can be rewritten as

$$
\min _{F} \hat{V}=\min _{\boldsymbol{F}} \operatorname{Tr}\left\{\overline{\mathbf{E}}^{*} \overline{\mathbf{E}} \mathbf{P}_{\boldsymbol{F}}\right\}
$$

where $\mathbf{P}_{F}=\mathbf{F}\left[\mathbf{F}^{*} \mathbf{F}\right]^{-1} \mathbf{F}^{*}=\mathbf{F F} \mathbf{F}^{*}$ is the projection onto the (full rank) columns of $\mathbf{F}$. Equation (11) can be written in a similar fashion by solving for the separable linear term B:

$$
\min _{\Psi} \hat{V}=\min _{\Psi} \operatorname{Tr}\left\{\overline{\mathbf{E}}^{*} \overline{\mathbf{E}} \mathbf{P}_{\overline{\boldsymbol{\Psi}} *}^{\perp}\right\}
$$


where

$$
\bar{\Psi}^{*}=\left[\begin{array}{c}
\mathbf{I} \\
\Psi^{*}
\end{array}\right]
$$

Thus, the two minimization problems have equivalent forms ${ }^{\dagger}$ since both are minimizations of the same functional form over rank $d$ projection matrices in $\mathbb{C}^{2 d \times 2 d}$.

To see that the matrix $\Psi$ obtained from (29) is identical to the ESPRIT estimate $\hat{\Psi}_{\mathrm{ES}}$, begin by defining

$$
\mathbf{E}_{1}=\left[\begin{array}{l}
\mathbf{E}_{11} \\
\mathbf{E}_{21}
\end{array}\right] \text { and } \mathbf{E}_{2}=\left[\begin{array}{l}
\mathbf{E}_{12} \\
\mathbf{E}_{22}
\end{array}\right]
$$

and note that minimizing (29) will lead to a projection operator satisfying $\mathbf{P}_{\mathbf{\psi}^{*}}^{\perp}=\mathbf{P}_{\mathbf{E}_{2}}$. By the orthogonality of the eigenvectors of $\overline{\mathbf{E}} * \overline{\mathbf{E}}, \mathbf{P}_{\bar{\Psi}^{*}}^{t}=\mathbf{P}_{\mathbf{E}_{2}}$ implies $\mathbf{P}_{\bar{\psi}^{*}}=\mathbf{P}_{\mathbf{E}_{1}}$. Therefore, there exists a non-singular matrix $\mathbf{T}$ such that

$$
\left[\begin{array}{c}
I \\
\Psi^{*}
\end{array}\right]=\left[\begin{array}{c}
\mathbf{E}_{11} \\
\mathbf{E}_{21}
\end{array}\right] \mathbf{T} \text {. }
$$

The estimate of $\Psi$ is thus given by $\mathbf{E}_{11}^{-*} \mathbf{E}_{21}^{*}$, and the orthogonality relation

$$
\mathbf{E}_{11}^{*} \mathbf{E}_{12}+\mathbf{E}_{21}^{*} \mathbf{E}_{22}=0
$$

now easily establishes the equivalence $\hat{\boldsymbol{\Psi}}=\hat{\boldsymbol{\Psi}}_{\mathrm{ES}}=-\mathbf{E}_{12} \mathbf{E}_{22}^{-1}$.

\section{Appendix B. Derivation of $Q$.}

In this appendix, the expression for the matrix $\mathbf{Q}=\mathscr{E}\left\{\left(\hat{V}^{\prime}\right)\left(\hat{V}^{\prime}\right)^{T}\right\}$ in $(23)$ is derived. The $i$ th element of $\hat{V}^{\prime}$ will be denoted by $\hat{V}_{i}^{\prime}$, and may be written as

$$
\begin{aligned}
& \hat{V}_{i}^{\prime \text { def }}=\left.\frac{\partial \hat{V}\left(\boldsymbol{\eta}_{0}\right)}{\partial \boldsymbol{\eta}_{i}}\right|_{\boldsymbol{\eta}=\eta_{0}}=\frac{\partial}{\partial \eta_{i}} \operatorname{Tr}\left(\mathbf{P}_{G}^{\perp} \mathbf{J} \hat{\mathbf{E}}_{s} \hat{\mathbf{E}}_{s}^{*} \mathbf{J}^{T}\right) \\
& =\operatorname{Tr}\left(\frac{\partial \mathbf{P}_{G}^{\perp}}{\partial \boldsymbol{\eta}_{i}} \mathbf{J} \hat{\mathbf{E}}_{\boldsymbol{s}} \hat{\mathbf{E}}_{\boldsymbol{s}}^{*} \mathbf{J}^{\boldsymbol{T}}\right) \\
& =2 \mathscr{R} e\left[\operatorname{Tr}\left(\mathbf{P}_{G}^{\perp} \mathbf{G}_{i} \mathbf{G}^{\dagger} \mathbf{J} \hat{\mathbf{E}}_{s} \hat{\mathbf{E}}_{s}^{*} \mathbf{J}^{T}\right)\right] \\
& \simeq 2 \mathscr{R e}\left[\operatorname{Tr}\left(\mathbf{P}_{G}^{\perp} \mathbf{G}_{i} \mathbf{G}^{\dagger} \mathbf{J} \mathbf{E}_{s} \tilde{\mathbf{E}}_{s}^{*} \mathbf{J}^{T}\right)\right] \text {. }
\end{aligned}
$$

The approximation of (B1) is obtained by substituting $\hat{\mathbf{E}}_{s}=\mathbf{E}_{s}+\tilde{\mathbf{E}}_{s}$ and dropping all second order error terms. The derivatives $\mathbf{G}_{i}=\partial \mathbf{G} / \partial \eta_{i}$ on which both $\hat{V}^{\prime}$ and $V^{\prime \prime}$ depend may be computed as follows:

$$
\begin{aligned}
& \text { 1. } \boldsymbol{\eta}_{i}=\mathscr{R} e\left\{\mathbf{A}_{k l}\right\} \\
& \mathbf{G}_{i}=\left[\begin{array}{c}
\mathbf{I}_{k l} \\
\mathbf{I}_{k l} \mathbf{\Phi}
\end{array}\right] \\
& \text { 2. } \boldsymbol{\eta}_{i}=\mathscr{I}_{m}\left\{\mathbf{A}_{k l}\right\} \\
& \mathbf{G}_{i}=j\left[\begin{array}{c}
\mathbf{I}_{k l} \\
\mathbf{I}_{k l} \mathbf{\Phi}
\end{array}\right]
\end{aligned}
$$

\footnotetext{
† There is, however, a subtle difference in the constraints. Mathematically speaking, the set of matrices of the form $\bar{\Psi}^{*}$ is a subset of all full-rank matrices in $\mathbb{C}^{2 d \times d}$ since full-rank matrices whose upper $d \times d$ block is not invertible can not be converted into the form of $\bar{\Psi}^{*}$. For the problems considered herein, such an event occurs with probability zero and need not be considered further.
} 
3. $\eta_{i}=\rho_{k}$

$$
\mathbf{G}_{i}=\frac{1}{\rho_{k}}\left[\begin{array}{c}
0 \\
\mathbf{A \Phi} \mathbf{I}_{k k}
\end{array}\right]
$$

4. $\eta_{i}=\theta_{k}$

$$
\mathbf{G}_{i}=-\left(j 2 \pi \Delta \cos \theta_{k} / \lambda\right)\left[\begin{array}{c}
\mathbf{0} \\
\mathbf{A \Phi} \mathbf{I}_{k k}
\end{array}\right]
$$

where $\mathbf{I}_{k l}$ denotes the matrix with 1 in the $k$, $i$ th position and zeros elsewhere.

The cost function gradient at $\boldsymbol{\eta}_{0}$ can now be written as a function of the array perturbation matrix $\tilde{\mathbf{A}}_{e}$ by substituting (20) into (B1):

$$
\hat{V}_{i}^{\prime} \simeq 2 \mathscr{R} e\left[\operatorname{Tr}\left(\mathbf{P}_{G}^{\perp} \mathbf{G}_{i} \mathbf{G}^{\dagger} \mathbf{J} \mathbf{E}_{s} \tilde{\mathbf{\Lambda}}^{-1} \mathbf{E}_{s}^{*} \mathbf{A}_{e} \mathbf{S} \tilde{\mathbf{A}}_{e}^{*} \mathbf{J}^{T}\right)\right] .
$$

Using the relationships $\mathbf{J E} \mathbf{E}_{s}=\mathbf{G T}, \mathbf{G}^{\dagger} \mathbf{G}=\mathbf{I}, \mathbf{E}_{s}^{*} \mathbf{A}_{e}=\mathbf{T}^{-1}$, and $\mathbf{S}=\mathbf{T} \tilde{\mathbf{\Lambda}} \mathbf{T}^{*}$, this equation can be rewritten in the somewhat simpler form

$$
\hat{V}_{i}^{\prime} \simeq 2 \mathscr{R} e\left[\operatorname{Tr}\left(\mathbf{P}_{G}^{\perp} \mathbf{G}_{i}\left(\mathbf{A}_{e}^{*} \mathbf{A}_{e}\right)^{-1} \tilde{\mathbf{A}}_{e}^{*} \mathbf{J}^{T}\right)\right]=2 \mathscr{R} e\left[\operatorname{Tr}\left(\left(\mathbf{A}_{e}^{*} \mathbf{A}_{e}\right)^{-1} \mathbf{G}_{i}^{*} \mathbf{P}_{G}^{\perp} \mathbf{J} \tilde{\mathbf{A}}_{e}\right)\right] .
$$

Since $\mathscr{E}\left\{\hat{\boldsymbol{\eta}}-\boldsymbol{\eta}_{\mathrm{o}}\right\}=\left(V^{\prime \prime}\right)^{-1} \mathscr{E}\left\{\hat{V}^{\prime}\right\}$, it is clear that to first order, there will be no estimate bias as long as the array errors are zero-mean (i.e., $\mathscr{E}\left\{\tilde{\mathbf{A}}_{e}\right\}=0$ ). To find a compact expression for $\mathbf{Q}$, define the quantity

$$
\mathbf{Y}^{(i)}=\left(A_{e}^{*} A_{e}\right)^{-1} G_{i}^{*} P_{G}^{\perp} J
$$

so that $\hat{V}_{i}^{\prime}$ may be rewritten as

$$
\hat{V}_{i}^{\prime}=2 \mathscr{R} e\left[\operatorname{Tr}\left(\mathbf{Y}^{(i)} \tilde{\mathbf{A}}_{e}\right)\right] .
$$

Using the fact that, for two complex numbers $\alpha$ and $\beta$,

$$
\mathscr{R} e\{\alpha\} \mathscr{R} e\{\beta\}=\frac{1}{2}\left(\alpha \beta^{*}+\alpha \beta\right),
$$

it is easily seen that

$$
\mathbf{Q}_{i k}=\mathbf{E}\left\{\hat{V}_{i}^{\prime} \hat{V}_{k}^{\prime}\right\}=2 \mathscr{R} e\left[\sum_{p=1}^{d} \sum_{q=1}^{d} \mathbf{Y}_{p}^{(i)} \mathbf{C}_{a, 1}^{p q} \mathbf{Y}_{q}^{(k) T}+\mathbf{Y}_{p}^{(i)} \mathbf{C}_{a, 2}^{p q} \mathbf{Y}_{q}^{(k)^{*}}\right]
$$

where $\mathbf{Y}_{p}^{(i)}$ denotes the $p$ th row of $\mathbf{Y}^{(i)}$ and

$$
\begin{aligned}
& \mathbf{C}_{a, 1}^{p q}=\mathscr{E}\left\{\tilde{\mathbf{a}}\left(\theta_{p}\right) \tilde{\mathbf{a}}^{T}\left(\theta_{q}\right)\right\}, \\
& \mathbf{C}_{a, 2}^{p q}=\mathscr{E}\left\{\tilde{\mathbf{a}}\left(\theta_{p}\right) \tilde{\mathbf{a}}^{*}\left(\theta_{q}\right)\right\},
\end{aligned}
$$

as in $\S 4$.

To arrive at (24), note that for the model of (15), if $\mathscr{E}\left\{\tilde{\mathbf{a}}\left(\theta_{i}\right) \tilde{\mathbf{a}}^{*}\left(\theta_{k}\right)\right\}=0, i \neq k$, and $\mathbf{B}_{k}=\mathbf{B}, k=1, \ldots, d$, then

$$
\begin{aligned}
& \mathbf{C}_{a, 1}^{p q}=0 \\
& \mathbf{C}_{a, 2}^{p q}=0, \quad p \neq q, \\
& \mathbf{C}_{a, 2}^{p p}=\mathbf{B}, \quad p=1, \ldots, d .
\end{aligned}
$$

In this case, the general expression of (B2) simplifies to

$$
\mathbf{Q}_{i k}=2 \mathscr{R} e\left[\sum_{p=1}^{d} \mathbf{Y}_{p}^{(i)} \mathbf{B} \mathbf{Y}_{p}^{(k)^{*}}\right]=2 \mathscr{R} e\left[\operatorname{Tr}\left(\mathbf{Y}^{(i)} \mathbf{B} \mathbf{Y}^{(k)^{*}}\right)\right] .
$$

Equation (24) can now be determined directly from (B3). 


\section{List of symbols}

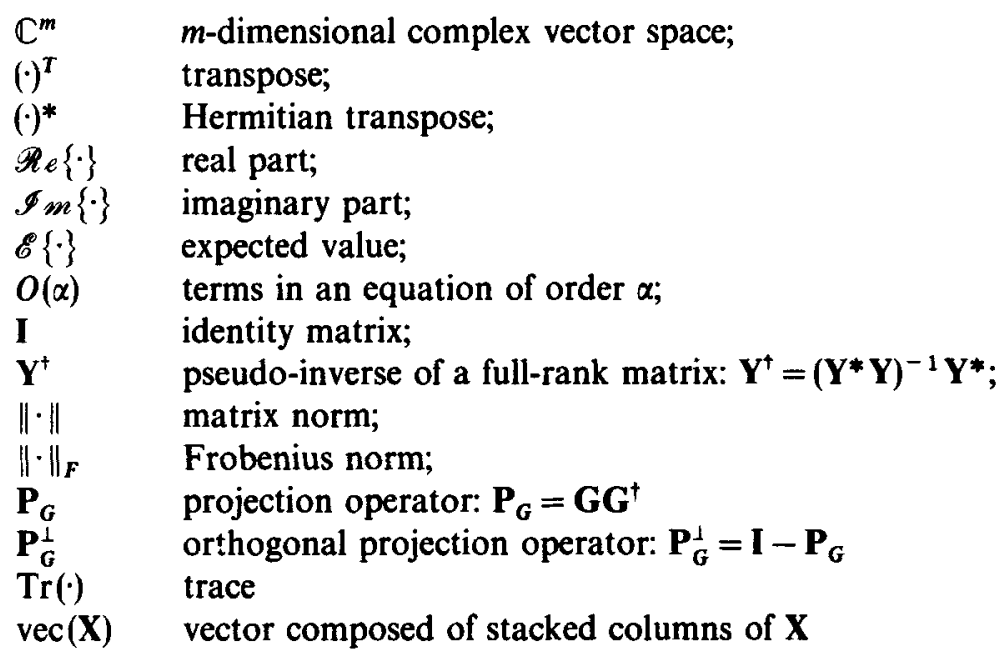

\section{References}

Golub G H, Van Loan C F 1984 Matrix comptutations (Baltimore, MD: Johns Hopkins University Press) Kung S Y, Lo C K, Foka R 1986 A Toeplitz approximation approach to coherent source direction finding. In Proc. IC ASSP 86 (Piscataway, NJ: IEEE)

Ottersten B E 1989 Parametric subspace fitting methods for array signal processing, PhD thesis, Stanford University

Ottersten B, Viberg M, Kailath T 1989 Asymptotic analysis of the total least squares ESPRIT algorithm. In Proc. SPIE Conf. on Advanced Algorithms and Architectures for Signal Processing IV (Bellingham, WA: Intl. Soc. Opt. Eng.) pp. 146-157

Ottersten B, Viberg M, Kailath T 1990 Performance Analysis of the Total Least Squares ESPRIT Algorithm. IEEE Trans. Acoust., Speech Signal Process. (accepted)

Paulraj A, Roy R, Kailath T 1986 A subspace rotation approach to signal parameter estimation. Proc. IEEE 74: 1044-1045

Rao B D, Hari K 1988 Performance analysis of subspace based methods. In Proc. 4th ASSP Spectral Estimation Workshop, pp. 92-97

Rao B D, Hari K V S 1989 Performance analysis of root-MUSIC. IEEE Trans. Acoust., Speech Signal Process. 37: 1939-1949

Rao B D, Hari K V S 1990 Performance analysis of ESPRIT, minimum-norm method and TAM in determining the direction of arrival of plane waves in noise. IEEE Trans. Acoust., Speech Signal Process. (in review)

Roy R H 1987 ESPRIT-Estimation of signal parameters via rotational invariance techniques, PhD thesis, Stanford University

Roy R, Kailath T 1989 ESPRIT - Estimation of signal parameters via rotational invariance techniques. IEEE Trans. Acoust., Speech Signal Process. ASSP-37: 984-995

Roy R, Paulraj A, Kailath T 1986 ESPRIT - A subspace rotation approach to estimation of parameters of cisoids in noise. IEEE Trans. Acoust., Speech Signal Process. ASSP 34: 1340-1342

Schmidt R 1981 A signal subspace approach to multiple emitter location and spectral estimation, PhD thesis, Stanford University

Swindlehurst A, Kailath T 1990 A performance analysis of subspace-based methods in the presence of model errors - Part 1: The MUSIC algorithm. IEEE Trans. Acoust., Speech Signal Process. (in review)

Swindlehurst A, Ottersten B, Kailath T 1989 An analysis of MUSIC and root-MUSIC in the presence of sensor perturbations. In Proc. 23rd Asilomar Conference of Signals, Systems, and Computers. pp. 930-934

Weiss A J, Friedlander B 1988 Direction finding in the presence of mutual coupling. In Proc. 22nd Asilomar Conf. on Signals, Systems, and Computers, 598-602 